\title{
Ki67: from antibody to molecule to understanding?
}

\author{
W Ross, P A Hall
}

The monoclonal antibody Ki67 was first described in 1983 by Johannes Gerdes and colleagues, who suggested that it might be used as a marker for proliferating cells. ${ }^{1}$ Over 10 years later, immunostaining with antibodies to the Ki67 antigen is well established as a quick tions of various tumour types because of its distinctive reaction patterns that exclusively involve proliferating cells. The extensive use of $\mathrm{Ki} 67$ as a marker of proliferation in pathology is evidenced by the large number of citations, which is increasing year on year. The Ki67 antibody was first isolated during attempts to raise monoclonal antibodies to antigens specific for Hodgkin and Reed-Sternberg cells. ${ }^{1}$ Ki67 stood out from other antibodies produced because it only reacted with cells which were proliferating, for example cortical thymocytes and cells in the crypts of the small intestine, whereas it would show no reaction with cells which were known to be in a resting or terminally differentiated state, such as liver cells and neurones. Furthermore there was good evidence that cycling cells in vitro also expressed the antigen. ${ }^{2}$ The pattern of $\mathrm{Ki} 67 \mathrm{ex}$ pression is not thought to be affected by DNA damage; for example, ultraviolet irradiation of cells in vitro and in vivo resulted in no visible alteration of $\mathrm{Ki} 67$ expression or localisation. ${ }^{3}$ Similarly, the induction of apoptosis appears to have no visible consequence upon $\mathrm{Ki} 67$ protein expression. ${ }^{4}$ The protein is constantly present in cycling cells at every stage of the cell cycle, although its presence in $G_{1}$ is variable. When postmitotic MCF-7 cells were analysed by fluorescence image flow cytometry there appeared to be several different patterns of Ki67 expression ${ }^{5}$ :(1) nucleoplasmic staining; (2) nucleolar staining; (3) finely speckled staining; (4) no staining at all (cells which have entered $\mathrm{G}_{0}$ ). These staining patterns were correlated with nuclear size to give three different types of progression through $G_{1}$ which could be characterised by the Ki67 expression:

Department of Pathology, University of Dundee, Dundee DD1 9SY

W Ross

P A Hall

Correspondence to: Ms Wendy Ross.

Accepted for publication 24 March 1994

(1) The Ki67 stable pathway-In this, Ki67 staining remains visible in the cells during $M$ $G_{1}$. The nuclear area was seen to be increasing in these cells.

(2) The Ki67 decrease pathway-The Ki67 staining becomes nuclear and then disappears. Small nuclei showed this pattern of expression. and efficient method for evaluating growth frac-

(3) The Ki67 increase pathway-The level of Ki67 staining is increasing to match that of the Ki67 stable pathway cells. Nuclear area also increased at this stage.

What do these different pathways represent? It could be that there are some cells which are capable of almost immediate entry into $S$ phase, requiring only a very short $G_{1}$. These cells may have a local environment which is supportive of this fast progression and so no downregulation of $\mathrm{Ki} 67$ expression occurs. It may be that those cells which show a decrease in Ki67 enter a quiescent period after mitosis in which they are sensitive to growth factors and mitogens and there is a downregulation of $\mathrm{Ki} 67$ expression. If the appropriate stimulation occurs, then progression through $G_{1}$ occurs and there is a subsequent increase in the level of Ki67 protein in the cell, that is, in those cells showing the "Ki67 increase pathway". If no stimulation to proliferate is received then the cell enters $G_{0}$ and production of the $\mathrm{Ki} 67$ protein drops to an undetectable level.

What is clear is that the expression of $\mathrm{Ki} 67$ in cells indicates that they are cycling. Furthermore the quantitation of the proportion of Ki67 expressing cells equates with the growth fraction of that population. ${ }^{67}$ Information on the growth fraction of tumours may be used in the assessment of tumour grade, and in all tumours which have been studied by $\mathrm{Ki} 67$ staining, a highly significant correlation between Ki67 staining and the degree of malignancy has been reported. Furthermore, a marked variation in the amount of Ki67 within different tumour grades is seen, indicating that Ki67 staining may be of use in individual tumour diagnosis and prognosis. ${ }^{8-10}$ The Ki67 protein has been shown to have an extremely short half life of around 20 minutes throughout the cycle, with major catabolism occurring at the end of mitosis, ${ }^{11}$ indicating that few cells which have left the cycle will contain the antigen.

\section{Ki67 and the nucleus}

In the early studies which showed that the Ki67 antigen is a cell proliferation related protein it was also noted that the intranuclear distribution of the antigen altered dramatically throughout the cell cycle. Further studies into the local- 
isation of Ki67 during the cell cycle by Verheijen et al in MR65 cells ${ }^{1213}$ and van Dierendonck et al in MCF-7 cells ${ }^{14}$ showed very clearly that the Ki67 protein has a very distinct localisation pattern:

Interphase-Predominant staining of the nucleoli, with diffuse nucleoplasmic staining.

Prophase-When chromosome condensation is not quite complete the staining becomes nucleoplasmic and begins to concentrate around the chromosomes.

Metaphase-A reticulate network of fibrils appears around the chromosomes, the staining becoming more granular in appearance. The staining of this network is uniform around the chromosomes.

Anaphase - The network persists around the chromosomes, becoming more granular in appearance. The density of the Ki67 staining is greatest at this point.

Telophase-As the daughter nuclei form and the chromosomes decondense, Ki67 staining becomes speckled throughout the nucleoplasm. The pattern then becomes nucleolar as the nucleoli also reform.

These very detailed observations raise questions about the function of the protein at different time points in the cell cycle and how these functions can be correlated with the distinct spatial pattern of expression with is seen. Of critical importance is the realisation that the alterations in expression pattern mirror in many respects the nucleolar cycle. ${ }^{1516}$

Nucleoli are familiar to all pathologists but their structure and function are less well understood. Nucleoli are non-membranous structures which occupy a large proportion of the nucleus. Within these organelles preribosomal components are synthesised and assembled, rRNA genes are transcribed by DNA dependent RNA polymerase I (also known as PolI), and processing of primary transcripts into mature rRNA occurs. They are proteinand RNA-rich structures which are made up of three main regions:

The fibrillar centre-This contains topoisomerase II, RNA polymerase I, and the transcription factor UBF.

The dense fibrillar compartment-This region almost completely surrounds the fibrillar centre. It is composed mainly of fibrillin, involved in ribosome synthesis.

The granular layer-Almost mature preribosomes are found in this region and are released from this point to move out into the cytoplasm.

The distinct localisation of Ki67 in the nucleoli during the $G_{1}$ and $S$ phases of the cell cycle suggests an association with the abundant RNA species found there. The Ki67 protein is localised mainly to the dense fibrillar compartment and the association appears to be extremely tight. The nuclear matrix is defined as "the biochemical entity which can be isolated after sequential extraction of cells with nonionic detergents, nucleases and high salt buffers". ${ }^{17}$ When nuclear matrix preparations of MR65 cells were made it could be seen that the $\mathrm{Ki} 67$ protein remained associated with the matrix and cytoskeletal component and was not removed along with the chromosomal material. Similarly, when nuclear matrix preparations of mitotic cells were made the antigen was still retained in the matrix in an identical distribution pattern to that seen in mitosis. These results in turn suggest a structural role for $\mathrm{Ki} 67$ in the nucleoli and during mitosis in association with the chromosomes.

As the cell progresses towards mitosis and cell division there are dramatic alterations in the structure of the nucleus-the nuclear envelope breaks down, the nucleoli disperse, and the chromosomes condense. The condensation of the chromosomes is probably the most crucial step in mitosis, being required for faithful dispersal of genetic material to each of the daughter cells. During this process, the DNA in each chromosome is condensed 100000 -fold until each is only a few microns in length. This strengthens the chromosomes such that they can withstand the great mechanical forces exerted upon them during metaphase and anaphase, but exactly how this condensation process is brought about is poorly understood. A network of proteins is believed to support the chromosomes in this condensed state - the chromosome scaffold-which can be isolated after removal of all histone proteins and most of the DNA from cells. Ki67 is found within this fraction, located around the chromosomes in a layer termed "the chromosome periphery". ${ }^{18}$ Numerous other proteins are found in this layer, many of which have relocated from the nucleoli to this region. Gautier et al reported a vast redistribution of nucleolar antigens around the chromosome surface in a confocal laser scanning microscopy study of mitotic cells from various mammalian species. ${ }^{19}$ This relocation seemed to occur in a highly ordered manner, mainly during prophase, with the proteins remaining associated until the end of telophase. A closer examination of this layer reveals that it consists of closely packed fibrils and dense granules. The granules are seen to be linked by thin filaments in some places. The layer is visible between sister chromatids but not in centromeric regions. The proteins found in this layer are not only nucleolar in origin: perichromin (a nuclear membrane associated protein), snRNAs, and snoRNAs, both nucleolar and nucleoplasmic in origin, are found in this layer. ${ }^{20}$ The functions of the layer are not clear but the proteins could be performing one of many roles, for example, forming a protective barrier around the chromosomes, helping to organise the chromosomes in their condensed state, or providing binding sites for chromosomal passenger proteins.

\section{The Ki67 protein}

The first genetic and biochemical data to emerge about the $\mathrm{Ki} 67$ protein resulted from attempts to clone and characterise the antigen by Gerdes et $a .^{21}$ This study showed that the Ki67 protein is very susceptible to proteases and had no properties in common with histone 
proteins. Western blotting of total cell lysates from proliferating cells saw a doublet of protein stained with molecular weights of $345 \mathrm{kDa}$ and $395 \mathrm{kDa}$ under reducing and non-reducing conditions. A lambda gt 11 cDNA library prepared from proliferating IM-9 cells was screened using the Ki67 antibody, resulting in 10 positive clones being obtained, eight of which contained an identical sequence of $1095 \mathrm{bp}$. Within this sequence, repetitive sequences of $300 \mathrm{bp}$ were found and each of these in turn contained a highly conserved $62 \mathrm{bp}$ element. Northern blotting of RNA from proliferating cells using the $1095 \mathrm{bp}$ sequence as a probe revealed species ranging in size from 7.5 to $9.5 \mathrm{~kb}$, whereas no bands could be detected in RNA isolated from quiescent cells. These data gave the first impressions of the Ki67 antigen and it appeared that an extremely large and unusually structured protein had been discovered. The gene was located to chromosome $11 \mathrm{q} 25 \operatorname{ter}^{22}$ and further cloning of the cDNA took until 1993 to complete. ${ }^{23}$ Two isoforms of messenger RNA were found for the protein-a species of 9768 bases and a species of 8688 bases. The cDNA sequence can be split into three main regions, as follows.

(1) The $5^{\prime}$ portion/N-terminus-The first portion of the gene is untranslated, having a CpG-rich island and two 24 bp direct repeats. There are 10 potential nuclear localisation signals (NLS), eight of which are bipartite. Exon 7 lies within this region. This exon can be spliced out of the protein, but the functional significance of this is as yet unknown. It is this activity which is thought to bring about the two isoforms of the protein seen on western blots-exon 7 is $1080 \mathrm{bp}$ long, encoding a protein of approximately $50 \mathrm{kDa}$.
(2) The central portion-This region of the gene is extremely unusual. It consists entirely of exon 13 of the gene, which is $6845 \mathrm{bp}$ or almost two thirds of the entire coding sequence. It contains 16 concatenated repeats, each having a consensus sequence of $366 \mathrm{bp}$ or 122 amino acids, termed "the Ki67 repeat". Within the Ki67 repeats lie highly conserved 66 bp or 22 amino acid elements, "the Ki67 motif", all of which have $72-100 \%$ identity to the concensus sequence (figure).

There are several amino acids which are almost totally conserved in every repeat: cysteine, position 8 (in every repeat apart from 7); glutamic acid, positions 38 and 101 (in every repeat); glycine, positions 30 and 85 (in every repeat); and leucine, position 103 (in every repeat).

Each Ki67 motif is likely to form an $\alpha$ helix with a highly amophilic character, that is, the charged residues lie down one side of the helix with the hydrophobic amino acids lying down the opposite face of the helix. The Ki67 motif is known to be the immunodominant epitope of the protein-when fusion proteins containing parts of the Ki67 protein were made and bacterially expressed, the Ki67 antibody only recognised those fusions containing the Ki67 motif. $^{2324}$

The cDNA sequence shows 40 weak and 10 strong PEST sites. These are sites which are rich in Proline, Glutamic acid, Serine and Threonine, and they target the proteins containing them for rapid degradation at the end of mitosis. Such sequences are found in proteins whose expression must be strictly controlled, for example D-type cyclins, E-type cyclins, and other proteins which carry out regulatory functions. ${ }^{25}$ The 10 strongest PEST sites are
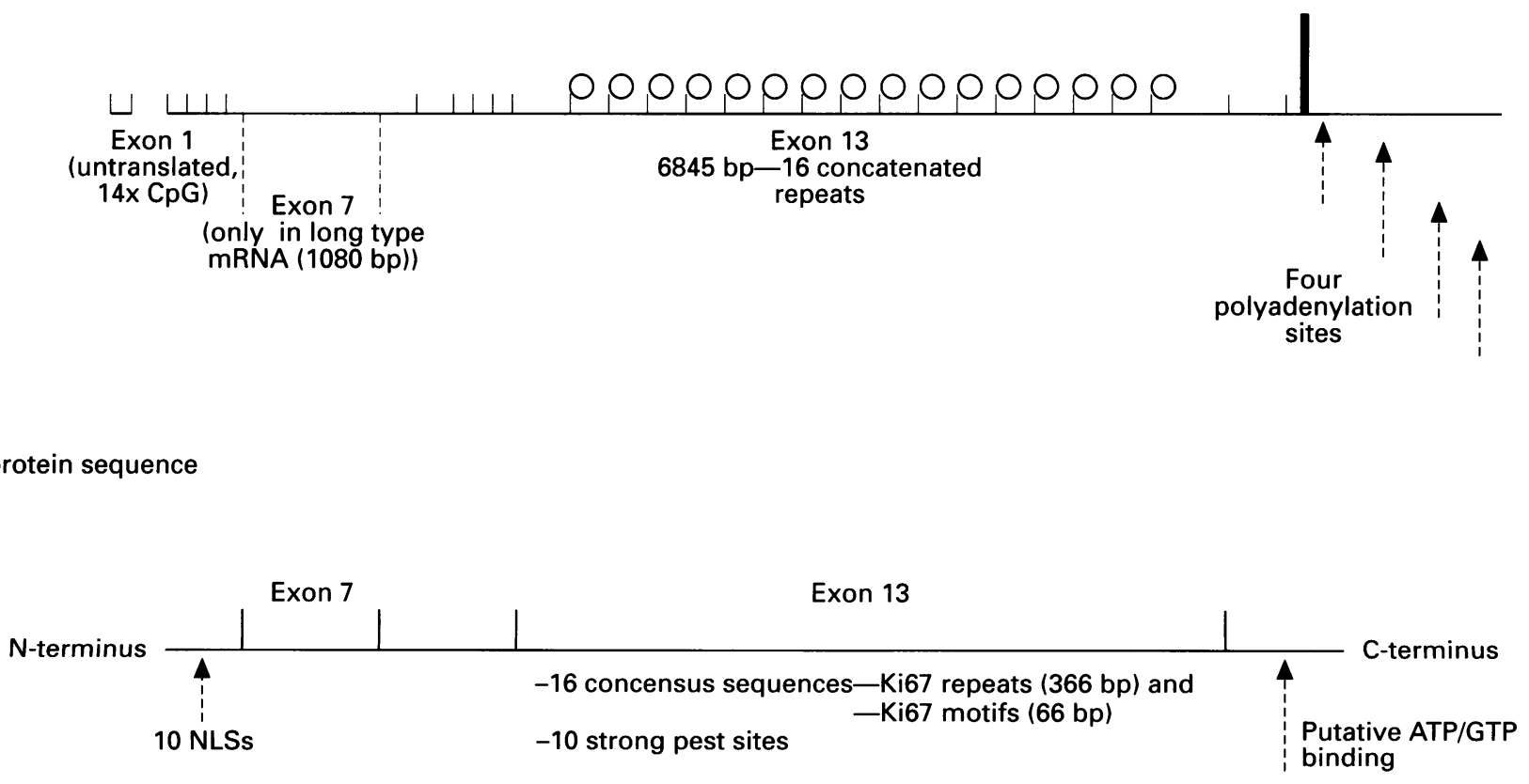

The Ki67 repeat (motif in bold, completely conserved residues underlined) 
found in exon 13, surrounding the highly conserved cysteine residues. The rapid loss of Ki67 protein after mitosis may be explained by the presence of these sequences.

(3) The 3' region/C-terminus-There is a putative ATP/GTP binding motif (a P-loop motif) at the extreme $\mathrm{C}$-terminus of the protein. This raises the possibility that $\mathrm{Ki} 67$ is in some way an active participant in cell cycle progression. This idea is further supported by the finding that antisense oligonucleotides specific to the first 20 bases adjacent to the ATG codon can inhibit ${ }^{3} \mathrm{H}$-thymidine uptake in IM-9 cells when concentrations of $5-20 \mu \mathrm{m}$ are used. ${ }^{23}$ The predicted protein sequence shows an enormous potential for post-translational modification, containing $19 \mathrm{~N}$-myristoylation sites, three amidation sites and over $200 \mathrm{pu}-$ tative phosphorylation sites. The sequence is rich in proline residues which suggests the protein may contain many turns and loops, with few $\alpha$ helices and $\beta$ sheets.

It has been shown that if DNA is present in cells or in nuclear extracts, Ki67 reactivity with its antigen increases. ${ }^{26}$ When cells were treated with DNAseI and then labelled with $\mathrm{Ki} 67$, the staining was reduced dramatically in a DNAseI concentration dependent manner. The Ki67 antigen seemed to protect DNA from the DNAseI treatment, as prestaining with $\mathrm{Ki} 67$ did not reduce the staining pattern to such a great degree. When nuclear extracts were mixed with DNA in vitro, dot blot analysis using the $\mathrm{Ki} 67$ antibody showed the DNA protein mixtures to be much more reactive than the nuclear proteins alone. When MIB 1 (another monoclonal antibody to the K67 motif) was used similar results were obtained, but it showed a greater affinity for protein alone that $\mathrm{Ki} 67$ showed for DNA Ki67 protein mixtures, and an even greater affinity for DNA protein mixtures. These experiments suggest that Ki67 may bind DNA, and that this activity may alter the protein such that it becomes much more immunoreactive. However no sequence specificity was found and further studies are therefore required to show a conclusive DNA:Ki67 interaction.

\section{Unanswered questions}

So what functions does this extremely unusual protein carry out within the cell? It is known that the antigen recognised by the Ki67 antibody is highly conserved throughout mammals, ${ }^{27}$ suggesting that at least the repeat structure is very important to its function. The predicted structure which is taken by these repeats is an amophilic $\alpha$ helix, which would correlate well with a nucleic acid binding role in either the nucleoli or in the perichromosomal layer. However, how 16 such helices would be orientated relative to each other is unclear. Indeed it is not certain that $\mathrm{Ki} 67$ always has 16 sets of repeats. Other proteins with repetitive structures, such as the mucins, show repeat number polymorphism. While that has not been demonstrated in $\mathrm{Ki} 67$, as yet there is no evidence that it does not happen. The $\mathrm{N}$-terminal and
C-terminal portion functions are also unclear. They could act as regulatory regions, responsible for controlling the activity of the protein. The protein has an enormous potential for regulation by phosphorylation, a universal cell cycle regulator, or by other forms of posttranslational modification. It is very tempting to imagine phosphorylation as a control both over the activity of the protein and perhaps also over its intracellular localisation. The size and unique nature of $\mathrm{Ki} 67$ make the challenge of unravelling its function considerable. A particularly attractive approach to understanding Ki67 will come from the cloning of homologues from other species, providing clues to conserved domains. Analysis of the function of such domains by overexpression strategies may also prove highly informative. The next few years should give us new insights into this fascinating molecule.

1 Gerdes J, Schwab U, Lemke H, Stein $\mathrm{H}$. The production of a mouse monoclonal antibody reactive with a human nuclear antigen associated with cell proliferation. Int $\mathcal{f}$ nuclear antigen associa

2 Gerdes J, Lemke H, Baisch H, Wacker HH, Schwab U, $\mathrm{Stein} \mathrm{H}$. Cell cycle analysis of a cell proliferation associated human nuclear antigen defined by the monoclonal antibody Ki67. F Immunol 1984;133:1710-8.

3 Hall PA, McKee PH, du P Menage H, Dover R, Lane DP. High levels of $\mathrm{p} 53$ protein in UV-irradiated normal human skin. Oncogene 1993;8:203-8.

4 Coates PJ, Hales S, Hall PA. The association between cell proliferation and apoptosis: studies using the cell cycle associated proteins Ki67 and DNA polymerase alpha. $f$ Pathol (in press).

5 du Manoir S, Guillard P, Camus E, Seigneurin D, Brugal G. Ki67 labelling in postmitotic cells defines different pathways within the 2c compartment. Cytometry 1991;12: 455-63.

6 McCormick D, Yu C, Hobbs C, Hall PA. The relevance of antibody concentration to the immunohistological quantification of cell proliferation-associated antigens. Histopathology 1993;22:543-7.

7 Scott RJ, Hall PA, Haldane JS, van Noorden S, Price Y, Lane DP, Wright NA. A comparison of immunohistochemical markers of cell proliferation with experimentally demarkers of cell proliferation with experimentally

8 Duchrow M, Gerdes J, Schluter C. The proliferation associated Ki67 protein: definition in molecular terms. Cell Prolif 1994;7:235-42.

9 Hall PA, Levison DA. Assessment of cellular proliferation in histological material. $\mathcal{F}$ Clin Pathol 1990;43:184-92.

10 Hall PA, Gregory W, Richards MA, d'Ardenne AJ, Lister TA, Stansfeld AG. The prognostic significance of Ki67 immunostaining in non-Hodgkin's lymphoma. 7 Pathol 1988;154:223-35.

11 Bruno S, Darzynkiewicz Z. Cell cycle dependent expression and stability of the nuclear protein detected by Ki67 antibody in HL-60 cells. Cell Prolif 1992;25:31-40.

12 Verheijen R, Kuijpers HJH, Schlingemann RO, Boehmer ALM, van Driel R, Brakenhoff GH, et al. Ki67 detects a nuclear matrix association proliferation related antigen. I. Intracellular location during mitosis. F Cell Sci 1989;92: 123-30.

13 Verheijen R, Kuijpers HJH, van Driel R, Beck JL, van Dierendonck JH, Brackenhoff GJ, et al. Ki67 detects a nuclear matrix associated proliferation related antigen. II. Localisation in mitotic cells and association with chromosomes. F Cell Sci 1989;92:531-40.

14 van Dierendonck JH, Keijzer R, van de Velde C, Cornelisse CJ. Nuclear distribution of the Ki67 antigen during the cell cycle: comparison with growth fraction in human cell cycle: comparison with growth fraction in him

15 Anastassova-Kristeva $M$. The nucleolar cycle in man. $f$ Cell Sci 1977;25:103-10.

16 Alberts B, Bray D, Lewis J, Raff M, Roberts K, Watson JD. Molecular biology of the cell, 3rd ed. New York: Garland Press, 1994.

17 Verheijen R, van Venrooij W, Ramaekers F. The nuclear matrix: structure and composition. $\mathcal{f}$ Cell Sci 1988;90: 11-36.

18 Hernandez-verdun D, Gautier T. The chromosome periphery during mitosis. Bioessays 1994;16:179-85.

19 Gautier T, Robert-nicoud M, Guilly M-N, Hernandezverdun $\mathrm{D}$. Relocation of nucleolar proteins around chromosomes at mitosis. A study by confocal laser scanning microscopy. F Cell Sci 1992;102:729-37.

20 Earnshaw WC, Bernat RL. Chromosomal passengers: toward an integrated view of mitosis. Chromosoma 1991; 100:139-46.

21 Gerdes J, Li L, Schluter C, Duchrow M, Wohlenberg C, Gerlach $\mathrm{C}$, et al. Immunobiochemical and molecular bio- 
logic characterisation of the cell proliferation associated nuclear antigen that is defined by monoclonal antibody Ki67. Am ₹ Pathol 1991;138:867-73.

22 Fonatsch C, Duchrow M, Reider H, Schluter C, Gerdes J. Assignment of the human Ki67 gene (MK167) to 10q25qter. Genomics 1991;11:476.

23 Schluter C, Duchrow M, Wohlenberg C, Becker MGH Key G, Flad H-D, et al. The cell proliferation associated antigen of antibody $\mathrm{Ki67}$ : a very large, ubiquitous nuclear protein with numerous repeated elements, new kind of cell cycle maintaining proteins. $f$ Cell Biol 1993;123:513-22.

24 Kubbutat MGH, Key G, Duchrow C, Flad H-D, Gerdes J. Epitope analysis of antibodies recognising the cell pro- liferation associated nuclear antigen previously defined by the antibody Ki67 (Ki67 protein). f Clin Pathol 1994;47: $524-8$.

25 Chevallier P. PEST sequences in nuclear proteins. (Review article). Int f Biochem 1993;25:479-82.

26 Lopez F, Belloc F, Lacombe F, Dumain P, Bernard P, Boisseau MR. The labelling of proliferating cells by Ki67 and MIB1 antibodies depend upon the binding of a nuclear protein to the DNA. Exp Cell Res 1994;210:145-53.

27 Falinin B, Flenghi L, Fagioli M, Stein H, Schwarting R, Riccardi $C$ et al. Evolutionary conservation in various Riccard $\mathrm{C}$, et al. Ev tutionary conservation mammalian species of the proliferation assocated epitope recognised by the Ki67 monoclonal antibody. $f$ Histochem
Cytochem 1989;37:1471-8. 